\title{
HISTOIRE ET SOCIÉTÉ
}

Jean-Claude Cheynet, Pouvoir et contestations à Byzance (963-1210). Éd. Centre de recherches d'histoire et de civilisation byzantines. Paris, Publications de la Sorbonne, 1990. $16 \times 24,523$ p., ill. en coul., index ( « Byzantina Sorbonensa ", 9).

L'histoire sociale de l'Empire byzantin a accumulé un certain retard par rapport à l'étude des autres champs de civilisation de la mềme époque; avec le livre de J.-C. Cheynet, elle le rattrape largement pour le sujet et la période considérés.

L'ouvrage donne, en effet, bien plus que le titre ne pourrait laisser croire. Bien entendu, ce que l'on attend - les révoltes et la réaction du pouvoir - s'y trouve, mais, al-delà, c'est un véritable portrait de la classe dirigeante byzantine que dresse l'auteur. Disons tout de suite que le lecteur qui chercherait les révoltes populaires sera quelque peu déçu; l'auteur étudie bien le peuple de Constantinople dans « les assises territoriales de l'opposition aux autorités », mais pour souligner la faible part qu'il joue, au moins comme initiateur, sinon comme masse de manouvre, surtout à partir de l'avènement des Comnènes. Le sujet mériterait peut-être d'être repris, notamment pour expliquer cette perte d'importance, en liaison avec la politique très hostile aux marchands et artisans menée par Alexis Comnène. Mais les sources sont, il faut le reconnaitre, indigentes sur le sujet, car les écrits, chroniques ou documents d'archives, sont le fait d'aristocrates, peu intéressés par la foule et qui la méprisent; quant aux autres types de sources, par exemple la sigillographie, dont $J . \leadsto$. Cheynet est un éminent spécialiste et qu'il utilise avec bonheur, ils proviennent par définition des fonctionnaires et des dignitaires laïs et ecclésiastiques. La signification péjorative du terme dêmokratia est d'ailleurs analysée de façon très judicieuse.

Le lecteur peut, en fait, se faire une idée de la documentation, car l'ouvrage commence par l'exposé systématique de celle-ci, à travers la fiche signalétique, dans l'ordre chronologique, de chacune des deux cent vingt-trois révoltes recensées, de l'avènement de Nicéphore Phocas à 1210. Quand il en dispose l'auteur présente les renseignements suivants : principaux protagonistes, famille d'origine, parenté impériale, objectifs de la rébellion, prédiction d'un destin impérial, actes de souveraineté accomplis par le rebelle, date, soutiens recueillis, opposants recensés, région touchée par la rébellion, sanctions encourues par celle-ci; chaque fiche se termine par les sources et la bibliographie. Immédiatement, J.-C. Cheynet rédige une sorte de vade-mecum du parfait révolté, la pratique de la révolte à Byzance, de la mise en place du complot à la propagande, du financement à l'intimidation, mais aussi la façon de sortir d'une révolte au moindre coût, les révoltes réussies étant finalement peu nombreuses. Notons au passage une remarque très importante : la révolte est un exercice qui nécessite un entraînement; il est rare qu'on réussisse du premier coup, sauf si l'on a déjà devant soi une importante expérience familiale. Avec beaucoup de modestie, ce vade-mecum est placé dans la partie adocu- 
mentaire " : on aura compris que ce développement est déjà une explication.

Les deux autres tiers de l'ouvrage se divisent entre « l'image et les fondements sociaux de la révolte » et une «interprétation des révoltes ». L'ordre est parfaitement logique, car l'auteur nous explique d'abord les bases idéologiques de la revolte et son substrat social. Le chapitre intitule «l'Empereur et le rebelle " est une mise au point précise sur la théorie et la pratique de la légitimité, qui montre l'adaptation aux circonstances du moment d'une idéologie qui remonte en fait aux premiers temps de l'empire chrétien : comment et pourquoi peut-on contester le choix de Dieu, en vertu duquel règnent les empereurs?

Puis J.-C. Cheynet fait un sort à l'explication que les auteurs byzantins euxmêmes donnent des crises, surtout au xI siècle, et que la bibliographie moderne a reprise avec eux : l'opposition entre civils et militaires. On sait que G. Ostrogorsky (cf. son Histoire de l'État byzantin) fondait ainsi son découpage de la période entre le régime de l'aristocratie civile et celui de l'aristocratie militaire; $P$. Lemerle, qui réfutait la vision «féodale » de cet auteur, ne s'en intéressait pas moins à la "démobilisation de l'Empire " (Cinq études sur le xf' siècle byzantin, p. 263-271) et mettait en avant cette opposition, chez les auteurs byzantins comme Michel Psellos, entre politikon génos et stratiôtikon génos, étant entendu que poliitkon désigne moins les civils en tant que tels que l'aristocratie constantinopolitaine. Or, J.-C. Cheynet démontre que cette opposition ne constitua nullement le ressort des luttes d'influence au $\mathrm{xI}^{\mathrm{e}}$ siècle : les familles passent d'un groupe à l'autre au gré de leurs intérêts et surtout comptent des représentants simultanément dans les deux groupes, qui ne sauraient donc être posés comme groupes sociaux antagonistes.

Ce phénomène constaté, il en donne une pertinente explication sociale, que l'on résumera de la façon suivante : de même que l'on ne peut distinguer vraiment l'aristocratie civile de l'aristocratie militaire, de même on ne peut opposer l'aristocratie constantinopolitaine à l'aristocratie provinciale. Bien évidemment, il existe des familles implantées de longue date à Constantinople et c'est chez elles que l'on exerce les hautes fonctions publiques de génération en génération; mais nombre de ces familles possèdent une importante fortune en province; réciproquement, les puissantes lignées provinciales s'implantent à Constantinople dès lors qu'elles veulent y exercer quelque influence; les noms des plus illustres familles de la capitale, souvent formés sur leur origine géographique, fournissent une démonstration irréfutable. Si l'on ajoute que l'on peut deviner des mariages, plutôt mal connus, l'aristocratie forme bien un seul ensemble, certes traversé de divisions et de contradictions, mais où l'on ne saurait opposer la capitale et la province. Bref, une famille aristocratique réside le plus souvent, au moins pour partie, dans la capitale, mais possède sa dotation foncière en province. Après 1204, évidemment, les choses changent, puisque ces familles, chassées de Constantinople, se réfugient sur ce qui reste de leur fortune foncière, après les chocs turc, normand et croisé.

J.-C. Cheynet complète alors son portrait de laristocratie byzantine par une étude de son "enracinement provincial ». Malgré le caractère lacunaire des sources, il parvient à des conclusions de grande importance, illustrées par trois cartes fort parlantes. On en résumera ainsi les résultats : les familles les plus puissantes, celles qui prétendent à l'Empire, ont leur base foncière dans la partie orientale 
de l'Asie Mineure, là même où elles exercent leurs commandements, et presque jamais en Europe; prudentes, elles ne s'installent pas dans les régions reconquises aux $\mathrm{x}^{\mathrm{e}}-\mathrm{xl} \mathrm{l}^{\mathrm{e}}$ siècles. L'implantation des familles secondaires est beaucoup plus diversifiée. L'avance turque contraint les familles les plus puissantes à réorienter leur base soit vers l'Europe, soit, surtout, vers l'ouest de l'Asie Mineure, ce qui leur permettra d'en sauvegarder une partie lors de l'épisode de l'Empire de Nicée.

Assuré de ces bases, l'auteur peut alors passer à une véritable définition de l'aristocratie byzantine, sujet dont l'intérêt dépasse de très loin l'histoire byzantine. Là encore, J.-C. Cheynet propose une interprétation nouvelle. Mème si les fonctions jouent un rôle dans la définition de l'aristocratie, même si un aristocrate se doit d'être riche, c'est la naissance qui est l'èlément essentiel; le renouvellement réel, mais limité, n'affaiblit nullement cette donnée de base, qui ne fait que se renforcer. L'importance d'une famille se mesure à l'éclat et au nombre de la parenté. À partir des Doukas et surtout des Comnènes, la parentèle participe en tant que telle au gouvernement. Les plus grandes familles de l'aristocratie déploient une stratégie d'alliances matrimoniales qui ne le cède en rien à celle de la famille régnante. Les Comnènes, particulièrement, ont su tisser à chaque génération un réseau de fidélités familiales impliquant presque toutes les grandes familles de l'Empire : le système politique repose alors au moins autant sur les alliances familiales que sur la notion de fonction publique. Certes, Byzance n'a pas vraiment connu de liens institutionnels d'homme à homme; mais les relations personnelles jouent un rôle décisif.

Cette question des relations personnelles est abordée ici d'une façon très nouvelle dans les études byzantines : «les formes de dépendance ». L'aristocrate, avec sa famille et ses serviteurs de tous ordres, mais aussi ceux qui, libres par ailleurs de tout lien de dépendance ou de parenté, se mettent au service d'un puissant en échange de sa protection, forme une "maison", un oikos. Ces "familiers ", que le chef de l'oikos consulte éventuellement avant de prendre une décision, sont rétribués non en terres, mais grâce à la redistribution des gratifications impériales et aussi par l'appui fourni pour accomplir une carrière de fonctionnaire et de dignitaire. Ces liens, que le droit byzantin ignore, ce qui a conduit l'historiographie moderne à les négliger, sont même souvent héréditaires des deux côtés. Le mot le plus approprié pour le dépendant est " homme " et il engage sa «foi ». J.-C. Cheynet, prudent, ne tire pas de conclusion comparatiste de ces données; mais il montre comment ces liens personnels s'insinuent peu à peu dans l'appareil de l'État et remplacent les liens auparavant évidents qui unissaient l'Empereur, simplement parce qu'il etait l'Empereur, et son fonctionnaire. Ajoutons que, dans le chapitre suivant, il montre les militaires partant à la guerre avec leurs hommes, des soldats qui sont leurs familiers; le phénomène de l'armée privée se développe. Il n'est d'ailleurs pas nouveau; déjà, au vi siècle, Justinien dénonçait, sans pouvoir réellement réprimer, les troupes de "doryphores" (porte-lance) que les puissants personnages des provinces utilisaient pour piller les biens des faibles en toute impunité (novelle 30 , de 535).

On notera d'ailleurs que cette structure en oikos se retrouve tout naturellement au plan économique pour désigner l'ensemble de la fortune d'un aristocrate, le plus souvent administrée depuis un palais de Constantinople. Les liens de dépen- 
dance, constatés aussi à l'intérieur de l'aristocratie et vis-à-vis des serviteurs, se retrouvent également dans une certaine mesure pour les paysans (cf. notre étude Les Hommes et la terre à Byzance du vr au xf siècle, Paris, Publications de la Sorbonne, 1992, qui constitue le volume suivant de la collection).

Au chapitre suivant, J.-C. Cheynet étudie les grands corps de l'État, en se limitant d'ailleurs à l'armée et à l'Église, done en laissant de côté l'administration civile, ce que l'on peut peut-être regretter, mais se justifie par le faible rôle joué par les membres de celle-ci dans la contestation du pouvoir. Là encore, i1 démonte les relations internes aux militaires, dégageant l'importance des groupes armés privés : les notables disposent de véritables milices. Il illustre son propos de tableaux dressés empereur par empereur, montrant que le souverain considéré nomme aux postes de commandement parents, serviteurs personnels et autres. Voici les précautions prises pour prévenir les coups d'État. Sur ce plan, le rôle du patriarche apparaît moins important que celui des prélats provinciaux.

A partir de cette solide analyse sociale du pouvoir et de la contestation, la troisième partie de l'ouvrage est consacrée à l'interprétation des révoltes, selon un ordre naturellement chronologique. Basile II, confronté à la révolte de l'aristocratie d'Asie Mineure, réussit d'autant plus efficacement à la réduire que l'essentiel de son effort de conquête, pour lequel il a besoin de l'aristocratie locale, se situe en Occident. La poussée turque accentue encore l'abaissement relatif de l'aristocratie micrasiatique. Alexis $\mathrm{I}^{\mathrm{er}}$ rétablit son autorité contre les contestataires effectifs puis éventuels en s'appuyant sur un système familial. L'examen des dissidences régionales qui ont émaillé le $\mathrm{xI}_{\mathrm{e}}^{\mathrm{e}}$ siècle permet de conclure à leur caractère local et, tout compte fait, secondaire ; elles sont souvent la réponse à l'affaiblissement d'un empire désormais incapable de défendre ces régions, qui restent en principe attachées à Constantinople, mais constatent qu'elles ne doivent compter, pour se défendre, que sur elles-mêmes. Sous les Comnènes, la dynastie contrôle correctement le centre, mais la défiance s'accroît dans les provinces : même si l'effort de reconquête et de contrôle semble avoir réussi, tous les éléments de l'éclatement sont en place, pour peu que l'autorité centrale s'affaiblisse, ce qui se produit de 1180 à 1204 . Cette époque constitue bien le résultat de l'évolution d'ensemble : le coup d'État d'Alexis Ange en 1195 marque la victoire de l'aristocratie occidentale, tandis que les autonomies provinciales s'affirment, avec les premières révoltes de l'aristocratie locale.

Dès lors, la chute de l'Empire face aux Croisés semble libérer ces forces centrifuges et offre aux dynastes locaux un triomphe apparemment facile : l'Empire se décompose en minuscules principautés, puisqu'il n'y a plus vraiment de pouvoir à contester. Pour autant, cette forme nouvelle de l'exercice de l'autorité ne parvient pas à s'affirmer : la tradition centralisatrice byzantine triomphe encore, au profit des états successeurs de l'Empire (Pont, Nicée, Thrace, Épire) et qui serviront de base à la reconquête de 1261 et à l'Empire de Trébizonde. Et les Paléologues retrouveront le réseau social établi sous les Anges. Même si J.-C. Cheynet a la prudence de ne pas s'aventurer aussi loin, l'on sent bien que la tradition et l'idéologie impériales empêchent une décomposition de type féodal.

L'auteur rassemble l'apport de son travail dans une vigoureuse conclusion. L'aristocratie est la seule source de contestation du pouvoir à Byzance et l'Empe- 
reur est, à deux exceptions près, un aristocrate ; le groupe est, au bout du compte, relativement fermé et la tentative d'élargissement du $\mathrm{xI}^{e}$ siècle a totalement échoué. Ce groupe comporte des dissonances et des hétérogénéités, mais l'opposition entre civils et militaires est pure illusion : les vrais clivages sont régionaux et l'évolution défavorise l'Asie Mineure, principalement les Cappadociens, au profit de l'Occident, notamment à l'avènement des Comnènes. La montée progressive des particularismes entretenus par l'aristocratie locale n'a toutefois pas eu raison, malgré 1204 , de la tendance unitaire et centralisatrice de l'Empire : c'est la proximité à l'Empereur, même si celle-ci devient avant tout familiale, qui définit le rang dans la hiérarchie.

J.-C. Cheynet n'a pas prétendu résoudre tous les problèmes que pose l'histoire sociale et politique des deux cent cinquante ans qu'il a étudiés; son titre, on l'a vu, est extrêmement modeste. Et, certes, il reste des zones d'ombre, avant tout imputables à l'insuffisance des sources, notamment sur une partie des corps intermédiaires. Mais, à partir de la contestation du pouvoir, l'ouvrage dresse un tableau complet de l'aristocratie byzantine, de la vie politique dans ses fondements sociaux et idéologiques. Il expose ainsi un lumineux portrait de l'originalité byzantine, la primauté de l'État et l'intégration au système d'État de la classe dirigeante, même si le principe de gouvemement de celui-là, et donc d'organisation de celle-ci, se modifie fortement, au profit des relations avec la famille impériale. Le pouvoir impérial devient ainsi dynastique et abandonne partiellement la tradition romaine de la magistrature et de la délégation d'autorité. On aura compris l'importance de l'ouvrage, et aussi la façon dont il s'inscrit dans les tendances les plus récentes de l'école historique française, notamment en matière d'histoire politique.

Michel KaPLAN.

Le Monde des chanoines : $X I^{f}-X I^{\mathcal{E}}$ siècle. Toulouse, Privat, 1989. $14 \times 18,406 \mathrm{p}$. («Cahiers de Fanjeaux », $n^{\circ} 24$ ).

Depuis 1965, le Centre de Fanjeaux, placé sous le patronage de l'Institut catholique et de l'Institut d'études méridionales de la Faculté des lettres de Toulouse-Le Mirail, organise des sessions d'études consacrées à l'histoire religieuse du Languedoc. Les "Cahiers de Fanjeaux " recueillent régulièrement depuis un quart de siècle les communications de l'annèe précédente. Saini Dominique en Languedoc (Toulouse, Privat, 1966) inaugura la série. Après avoir exploré le monde des réguliers, les ordres monastiques et mendiants, la dernière session s'ouvre au "monde des chanoines" sous la présidence de Joseph Avril et avec la collaboration de dix-sept spécialistes.

Le titre s'applique parfaitement à la diversité de la vie canoniale dont l'étude impose d'élargir le champ d'investigation habituellement attaché aux xuI et $\mathrm{XJV}{ }^{2}$ siècles. La tradition est ancienne. Liée à la vie des diocèses, elle a été bouleversée par les réformes imposées par la centralisation et adaptée dans des regroupements nécessités par la déconcentration. Les chanoines sont des cleres inscrits sur la matricule d'une église. Ils appartiennent à la catégorie spéciale de clercs dis- 
tincte des moines, lesquels forment l'ordo monasticus vers la fin du vili ${ }^{\mathrm{e}}$ siècle. Voulant contrôler le mode de vie de ceux qui célèbrent les heures canoniales, font du ministère auprès des fidèles et ont en charge des missions d'assistance, Louis le Pieux, sous l'influence de Benoit d'Aniane, les soumet à la Regula canonicorum (synode d'Aix-la-Chapelle de 816) pour mieux marquer leur identité et leur spécialisation. Les chanoines ont subi ensuite l'influence des grandes réformes monastiques et ont défini des observances parallèles.

Le $\mathrm{XI}^{e}$ siècle est un siècle de réformes. Déjà attirés par les courants érémitiques dans la recherche de la vie des Apôtres envisagée essentiellement comme une vie religieuse communautaire, les milieux canoniaux multiplient les formes inédites. La lettre 2 I 1 d'Augustin (sous sa forme originale ou augmentée de divers compléments) est promue Regula sancti Augustini équivalente de la Regula sancti Benedicti pour les chanoines réguliers qui veulent former un ordo canonicus. Mais la nouvelle voie n'a pas séduit la masse des chanoines d'ancien régime réformés au début $d u{ }^{e}{ }^{e}$ siècle, lesquels ont suivi une évolution particulière.

L'étude du « monde des chanoines », qui commence par une introduction de Marie-Humbert Vicaire (p. 7-12), est limitée au Languedoc. Elle prend ainsi un relief saisissant, dont les grands traits avaient été esquissés par Élisabeth MagnouNortier ${ }^{29}$.

Dans les diocèses languedociens, les chapitres cathédraux et les collégiales (première partie) renforcent leur emprise : les uns ont conquis le droit d'élection exclusif des évêques, les autres ont contribué à encadrer la vie religieuse (p. 19 . 163). Dom Jean Becquet avait réuni ses recherches sur les chanoines dans la Vie canoniale en France aux $x^{c}$-xIf siècles ${ }^{30}$ et dressé l'état des questions dans "Vingt-cinq ans d'études canoniales en France (1959-1984) " ${ }^{31}$. II jalonne ici "l'évolution des chapitres cathédraux : régularisations et sécularisations ": un groupe de clercs encouragés par l'évêque élimine les séculiers du chapitre et joue un rôle prépondérant dans l'élection épiscopale avec le soutien du Siège apostolique. Le mouvement inverse vers la sécularisation commence au début $\mathrm{du}$ XIII ${ }^{e}$ siècle, avec la symétrique influence de la papauté. La régularité laisse néanmoins son empreinte à Arles, Carcassonne, Toulouse, Nîmes, Maguelonne jusqu'aux $\mathrm{xv}^{t}-\mathrm{xvr}^{\mathrm{e}}$ siècles (p. 19-39). Avec une riche documentation (les décrets conciliaires et les statuts synodaux, les documents épiscopaux et pontificaux), Joseph Avril aborde l'aspect fonctionnel de «la participation du chapitre cathédral au gouvernement du diocèse " et retrouve la question de la régularité. Maguelonne est exemplaire : l'évêque y est membre du chapitre, tenu comme celui d'un monastère. À l'instar des grandes abbayes, les chapitres cathédraux de Maguelonne, Carcassonne et Narbonne gèrent des réseaux de dépendances

29. Cf. « II. La réforme des clergés séculier et régulier : 1. L'adoption de la vie régulière par les communautés canoniales ", in La Société lä̈que et l'Église dans la province ecclésiastique de Narbonne (Zone cispyrénéenne) de la fin du nif à la fin du X广 siècle, Toulouse, 1974 ("Publications de l'Université de Toulouse-Le Mirail ", série A, t. 20), p. 475-490.

30. Londres, Variorum Reprints, 1985.

31. Cf. Liber amicorum. Études historiques offertes à Pierre Bougard, Arras, 1987 (Mémoires de la Commission départementale d'histoire et d'archéologie du Pas-de-Calais, t. XXV, Revue du Nord, hors série $\left.n^{\circ} 3,1987\right)$, p. 65-71. 
confiées à des prieurs pour une mission pastorale (p. 41-63). Marthe Moreau montre comment, à Béziers, l'évêque et le chapitre contrôlent « les chanoinesses du Saint-Esprit " (p. 105-116). Jean-Louis Biget exploite la documentation de la situation conflictuelle d'Albi, retrouve la chronologie du recrutement déjà jalonnée et suit l'évolution des rapports d'influence entre les deux institutions, "Sainte-Cécile et Saint-Salvi. Chapitre et collégiale ", dans la gestion du diocèse (p. 65-104). Jean-Loup Lemaitre explique comment « les obituaires des chapitres cathédraux du Languedoc au Moyen Âge » peuvent enrichir non seulement la connaissance de la liturgie mais aussi celle de la vie quotidienne des hommes et des institutions (p. 117-149). Dans « les constructions canoniales des chapitres cathédraux du sillon rhodanien et du littoral méditerranéen au temps de la Réforme grégorienne ", Yves Esquieu retrouve l'organisation de la vie régulière sans pouvoir généraliser (p. 151-163).

$\mathrm{Au} \mathrm{XI}{ }^{e}$ et au XII ${ }^{e}$ siècle, des cleres recherchent la sensibilité des temps apostoliques. Saint-Ruf est le premier grand mouvement à concrétiser l'ordo novus : le $1^{\text {er }}$ janvier 1039, quatre clercs de Notre-Dame d'Avignon s'installent dans l'église consacrée au premier animateur de la communauté des chrétiens d'Avignon que leur donne l'évêque. En 1059, Nicolas II (Gérard avait été évêque de Florence) oriente la nouvelle spiritualité des clercs (synode de Rome). Il leur demande de rechercher cette "vie commune", groupés en communautés dans leurs églises. Dans la lettre qu'il adresse en 1092 à l'abbé Arbert et aux chanoines de Saint-Ruf, Urbain II (disciple de l'écolâtre Bruno, Eudes de Châtillon (Châtillon-sur-Marne, ancien diocèse de Soissons) avait été chanoine à Reims avant d'entrer à Cluny; Grégoire VII l'en fit sortir pour le placer sur le siège d'Ostie) ne propose pas la restauration de l'Ordre, bien qu'il évoque, dans un long préambule, la rénovation de la vie des Pères et la doctrine apostolique. Il explique que " deux vies sont instituées pour ses fils depuis les origines de la sainte Église : l'une, que garde la pusillanimité des faibles, l'autre, que la bienheureuse vertu des forts rend florissante; l'une, qui reste dans sa petitesse à Coar [ville au sud-est de la mer Morte], l'autre, qui s'élève vers les hauteurs de la montagne; l'une, qui rachète par les larmes et les aumônes les péchés de chaque jour, l'autre, qui recherche par la persévérance de chaque jour les mérites éternels; ceux qui s'en tiennent au genre de vie infërieur usent des biens terrestres, ceux qui suivent la vie supérieure méprisent les biens terrestres et les abandonnent. Et celle qui se détoume du monde par la ferveur divine se partage en deux branches d'un propositum presque identique, celui des chanoines et des moines : la seconde, pratiquée de par la miséricorde divine, brille suffisamment déjà au siècle tout entier, mais la première, de par la ferveur décroissante des fidèles, a dégénéré presque complètement; Urbain, martyr et pontife, l'a fondée, Augustin l'a organisée par ses règles, Jérôme l'a décrite dans ses lettres. C'est pourquoi il ne faut pas estimer d'un moindre mérite de susciter cette vie primitive de l'Église inspirée et accompagnée par l'Esprit divin, que de garder la florissante vie religieuse des moines par la persévérance de ce même Esprit ». En conséquence, le pape reconnaît le propositum des chanoines de Saint-Ruf en lui donnant son autonomie. Le rapprochement des deux voies introduit ainsi la stimulante évocation de cette vita canonica. Huit ans plus tôt, deux chanoines de Saint-Ruf avaient accompagné Bruno et l'évêque Hugues dans les montagnes de Chartreuse. 
La deuxième partie est consacrée aux " ordres de chanoines" (p. 167-254). Elle s'ouvre naturellement sur Saint-Ruf, avec les contributions d'Yvette Lebrigand sur les « origines et [la] première diffusion de l'Ordre de Saint-Ruf » (p. 167179), de Pierre-Marie Gy sur « la liturgie des chanoines réguliers de Saint-Ruf » (p. 181-191) et de Pedro R. Rocha sur « le rayonnement de l'Ordre de Saint-Ruf dans la péninsule ibérique d'après sa liturgie " (p. 193-208). Henri Barthès relève dix-neuf " abbayes de Prémontré en Languedoc » dans la future circarie de Gascogne. Sainte-Marie de la Case-Dieu (Beaumarchés dans le Gers), la première fondation, essaime vers les Pyrénées (1135). L'implantation est particulièrement dense dans le Pays basque. Trois maisons prennent part aux controverses antivaudoises : Bernard $(\dagger 1193)$, premier abbé de Sainte-Marie de Fontcaude (diocèse de Narbonne; Cazedarnes dans l'Hérault), rédige un mémoire contra Valdenses et Arianos (1090/1092) pour « instruire les clercs, corriger ceux qui, ignorants ou manquant de livres, cèdent à l'erreur, au scandale des fidèles qu'ils dirigent "; son action est relayée à Saint-Laurent de Combelongue (diocèse de Couserans; Rimont dans l'Ariège) par l'abbé Navarre d'Acqs (élevé à l'épiscopat en 1208); Jean ( $†$ 1215), abbé de Sainte-Marie de La Capelle (diocèse de Toulouse; Merville en Haute-Garonne), combat l'hérésie par la prédication (p. 209236). "L'Ordre canonial et hospitalier des Antonins ", qu'étudie Daniel Le Blévec, est né autour des reliques miraculeuses d'un ermite sollicité principalement pour la guérison du mal des ardents, la communauté de frères, spécialisée dans les soins, ayant réussi à rejeter la tutelle des moines, chargés de l'hospitalité dans le prieuré rattaché à Saint-Pierre de Montmajour (p. 237-254).

$\mathrm{Au} \mathrm{XIII}^{\mathrm{e}}$ siècle, le modèle est toujours évangélique. Mais la pauvreté moderne n'est plus la pauvreté communautaire des Apôtres : c'est désormais la pauvreté volontaire dans l'abandon à la Providence. La mutation est proposée par un chanoine régulier de la cathédrale castillane d'Osma, saint Dominique, « co-chanoine " de Saint-Paul de Narbonne et de Saint-Nazaire de Carcassonne. Sous le titre «Idéal et réalités », la troisième partie regroupe des contributions qui recherchent les forces spirituelles ayant conduit à la régularisation d'un grand nombre de chapitres et de collégiales (p. 257-362). Est-il possible d'évoquer la spiritualité des chanoines? La prédication donne un éclairage en relief. Dans cette perspective, les sermons sont une source précieuse. Jean Longère évoque « les chanoines réguliers d'après trois prédicateurs du xIII ${ }^{e}$ siècle : Jacques de Vitry $(\dagger 1240)$, Guibert de Tournai $(\dagger 1284)$, Humbert de Romans $(\dagger 1277) »$ et leurs sermons ad status. Dans l'un des quatre sermons analysés ${ }^{32}$, le premier prédicateur évoque les origines sur le modèle du fleuve sorti de l'Éden, "qui a coulé jusqu'au bienheureux Augustin [lequel] a commencé à vivre selon la regula établie sous les saints apôtres". Le fleuve a donné sept bras, "sept congrégations de chanoines qui ont même regula et même fondamentum, mais des instituta différents : l'un des bras, les chanoines de Prémontré, l'autre, le convent de Grandmont, le troisième, l'Ordre de Saint-Victor, le quatrième, l'Ordre d'Arrouaise, le cinquième, l'Ordre du Val-des-Écoliers, le sixième, l'Ordre du Val-des-Choux, le sep-

32. Cf. «Quatre sermons ad canonicos de Jacques de Vitry », Recherches augustiniennes, t. XXIII, 1988, p. 151-212. 
tième, l'Ordre des Frères prêcheurs ". Le second prédicateur insiste sur la connaissance de l'Écriture qui donne la compréhension des rapports sociaux. Humbert de Romans, lui, fait l'éloge comparatif de la Règle de saint Augustin : liée à la vita apostolica, elle est plus ancienne, son autorité est supérieure (p. 257. 283). Les " maitres de Saint-Ruf " véhiculent la nouvelle culture juridique avant le milieu du XII e siècle. Sous le titre «les chanoines et les universités », Jacques Verger envisage l'influence du monde des chanoines dans le renouveau intellectuel et propose la statistique (faussée par le système bénéficial) des chanoines inscrits dans les universités méridionales au XIV siècle (p. 285-307). "Chanoines de chapitres méridionaux à la lumière des documents pontificaux d'Urbain V (13621372) ", la contribution d'Anne-Marie Hayez révèle les effets néfastes d'un tel système (p. 341-362). Avec « Benoît XII, Arnaud de Verdale et la réforme des chanoines ", Franz Josef Felten explique les circonstances de l'échec de la mission confiée par le pape de "réformer tous les chanoines de la province de Narbonne " (1335), les ressources des chapitres étant la chasse gardée des protégés bien introduits en cour (p. 309-339).

Le monde des chanoines est un monde à part, qui doit encore être exploré par des études ponctuelles ou générales, locales et régionales. Dans sa conclusion (p. 363-373), le président de la session, Joseph Avril, dresse le bilan et indique des perspectives de recherches fructueuses.

Jacques Foviaux.

Mouza Raskolnikov, Des Anciens et des modernes. Articles réunis par Ségolène Demougin, av.-pr. de Claude Nicolet. Paris, Publications de la Sorbonne, 1990. $16 \times 24,193$ p. ("Série Histoire ancienne et médiévale », 23, Université de Paris-I).

Ce livre est un recueil d'articles consacrés à l'historiographie du monde romain. Claude Nicolet souligne que l'historiographie est une discipline difficile, qui redouble les difficultés propres à la recherche historique, en obligeant l'historien à effectuer un continuel va-et-vient entre le monde romain et ceux qui l'analysent. M. Raskolnikoff étudie successivement deux lectures de l'histoire romaine : celle des historiens soviétiques au $\mathrm{xx}^{\mathrm{e}}$ siècle, et celle qui a été effectuée en France au XVIII siècle, pendant et juste après la Révolution.

Les recherches réalisées par les historiens soviétiques sont analysées en prenant en compte quatre paramètres. Analyse méthodologique : quelle influence ont pu jouer les travaux de certains historiens ou l'introduction de nouvelles méthodes comme la construction de modèles mathématiques? Rôle de la planification des recherches : comment les historiens soviétiques des années 60 ont-ils été conduits à s'intéresser essentiellement au problème de l'esclavage dans les sociétés antiques? Étude des concepts utilisés comme instruments d'analyse : comment les définitions de la révolution sociale, des modes de production, de l'importance à accorder à l'histoire politique ont-elles fourni des instruments d'interprétation de 
l'histoire romaine? Enfin, analyse de l'histoire même des Soviétiques : comment a-t-elle pu fournir des grilles de lecture pour percevoir l'Antiquité ? M. Raskolnikoff montre ainsi que la lecture de l'alliance des esclaves avec les paysans pauvres dans l'Antiquité est une transposition de l'alliance des prolétaires avec les paysans pendant la révolution d'Octobre.

L'étude effectuée au xvir ${ }^{c}$ siècle fait apparaître la grandeur et la décadence du mythe romain. Autant ce mythe a joué un rôle essentiel pendant la Révolution, rôle que l'on peut éclairer en analysant le systeme éducatif, les volontés d'identification et l'imaginaire collectif́, autant il a été dénoncé par le mouvement des Idéologues et, en particulier, par Volney. Volney associe sa critique de Rome à son refus de toute religion : les révolutionnaires ont substitué à la superstition juive la superstition chrétienne et ont ainsi contribué à développer l'affectivité et l'irrationalité. Volney dénonce toutes les violences, les injustices et les cruautés dont est pleine l'histoire des Grecs et des Romains, utilisant ainsi l'Antiquité pour confirmer sa thèse d'une perfectibilité indéfinie de l'espèce humaine. Critiquant les thèses de Rousseau et de Mably, Volney insiste sur les différences profondes qui séparent le xvil siècle de la Rome antique : il montre que la liberté des modernes ne peut se fonder que sur les réalités économiques. L'histoire romaine, privée de sa dimension normative, peut ainsi devenir un sujet d'études : Fustel de Coulanges en écrivant La Cité antique se réclame expressément des Idéologues.

Barbara de Negron],

Jacques Rossiadd, La Prostitution médiévale. Préf. Georges Duby. Paris, Flammarion, $1988.14 \times 22,288 \mathrm{p}$. (" Nouvelle bibliothèque scientifique $)$ ).

L'ouvrage rassemble un article paru en 1976 dans Annales. E.S.C., un essai écrit en 1984 et cinquante-trois pages de témoignages et de pièces justificatives, le tout essentiellement centré sur le sud-est de la France et l'Italie.

L'avertissement preliminaire replace le livre au milieu des dernières et plus importantes recherches en histoire sociale ; en effet, l'étude de la prostitution permet de compléter l'histoire de la sexualité en faisant, en quelque sorte, le lien entre l'étude de la morale et celle des comportements démographiques. Il fait également ressortir l'intérêt du choix de la région et de la période : de la Bourgogne à la Provence, tous les quartiers de rivière des villes rhodaniennes recèlent des « maisons » et, entre 1440 et 1490, cet ensemble géographique a été très peu touché par la guerre.

L'ensemble de l'étude montre combien la société de la fin du Moyen Âge considérait l'existence des bordels comme non seulement normale, mais aussi utile : les jeunes gens, à partir de $18-20$ ans, se rendent au prostibulum où "nature les meust d'aller "; c'était une preuve qu'ils ne séduisaient pas des filles ou des épouses, qu'ils n'entretenaient pas une femme. Ainsi, vers 1460, lorsque le marchand lyonnais François Garin compose une très longue complainte versifiée «pour l'éducation de ses fils", suggère-t-il que les bordels et les étuves 
accomplissent une ceuvre "selon nature " avant l'orientation vers le mariage. $\mathrm{Ce}$ devait être effectivement un exutoire aux violences sexuelles dont le nombre est élevé : Jacques Rossiaud relève 125 cas de viols connus entre 1436 et 1486 et pense qu'il s'agit du $1 / 5$ ou du $1 / 4$ de ce qui s'est réellement passé, $80 \%$ de ces

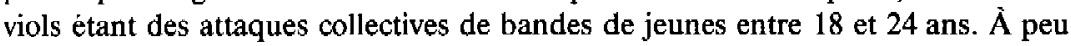
près tout le monde pensait que, face à cette violence, les filles communes sécurisaient la collectivité en contribuant, par leur existence, à défendre l'honneur des femmes d'estat. C'est pourquoi les conseils urbains et les pouvoirs territoriaux sont très permissifs, interdisant l'ouverture des maisons seulement pendant le temps de la messe, le dimanche, et celui de la Passion, avant Pâques : l'Église considère donc, en gros, leur existence comme un moindre mal.

Tout commence à changer à partir des années 1480-1500, époque à partir de laquelle les salaires urbains s'èrodent inexorablement, entraînant un cortège de misères et l'augmentation très sensible des effectifs de la prostitution; en mème temps, dans certaines villes, les filles de la bourgeoisie marchande obtenaient, plus ou moins, le libre choix de leur conjoint... La réforme des mours, comme le montre bien J. Rossiaud, passait donc par le cantonnement des filles publiques, d'une part, et la remise en ordre de la famille par la mise au pas des filles trop libres. On voit alors des municipalités fermer les étuves, imposer - comme au $\mathrm{XIII}^{\mathrm{e}}$ siècle - le port d'insignes distinctifs aux prostituées... On vit aussi l'hostilité aux «filles » monter parmi les «femmes de bien ", mais surtout on vit les prédicateurs se déchaîner et les juristes se mettre à la tâche. La répression fut lente mais continue, aboutissant vers 1560 à un changement très net de mentalité, où se mêlent l'austérité intransigeante des protestants et l'attitude rigoureuse des courants réformistes catholiques.

Cette belle étude fait ressortir avant tout la grande liberté masculine en matière de sexualité au $\mathrm{Xv}^{\mathrm{E}}$ siècle, les prostituées n'étant, dans leur immense majorité, que des victimes de la misère ou de la solitude considérées comme utiles... Mais cette liberté de mours masculine ne survécut pas en totalité aux nouvelles manières de penser qui, pourtant, ne supprimèrent pas la prostitution et entraînèrent un intense mépris envers les prostituées.

Françoise BONNEY.

Aeneas Silvius Piccolomini, Epistola ad Mahomatem II (Epistle to Mohammed II). Ed. with transl. and notes by Albert R. BACA. New York/Berne/Francfort-sur-le-Main/Paris, Peter Lang, 1989. 15,5 × 22,5, 223 p., bibliogr., index (« American University Studies ", ser, 2, " Romance Languages and Literature », vol. 127).

En 1453, les Turcs se sont emparés de Constantinople. Lorsque l'humaniste Aeneas Silvius Piccolomini devient le pape Pie II, il tente d'organiser une croisade, dont sa mort empêchera la mise en cuvre. Mais en même temps, il essaie un autre mode d'action : il rédige vers 1460 une longue lettre au Sultan pour le 
convaincre de ne pas attaquer I'Italie et, surtout, pour lui indiquer un moyen plus sûr d'étendre son pouvoir - la conversion au christianisme. L'essentiel du texte est done consacré a une confrontation des deux religions. C'est l'époque aussi de la Cribraito Alchorani de Nicolas de Cues (dont l'édition critique par L. Hagemann est parue en 1986 dans les Opera de l'Académie de Heidelberg; traduction en cours, Hagemann/Glei, dans la « Philosophische Bibliothek » de F. Meiner) et du Contra principales errores perfidi Machometi de Juan de Torquemada : sous des formes diverses, la confrontation theorique est à l'ordre du jour.

A vrai dire, comme le rappelle A. Baca, l'Epistola est plus proche du relevé d'erreurs que de l'analyse philosophique de la Cribratio, qui s'attache en outre à déceler comme des germes de christianisme dans le Coran. Le chapitre viI, en rattachant l'Islam à Arius et Nestorius, semble reprendre un argument cusain, mais l'essentiel consiste à énumérer des démarcations : la Trinité; le Sacrifice du Christ; la conception de l'Au-delà ; le statut de l'Écriture Sainte. Cependant l'ensemble, peut-être dans la mesure où il renonce à l" " interprétation pieuse » et à la "manuductio» qui marquent la méthode du cardinal de Cues, reflète-t-il mieux l'horizon oủ l'Islam désormais est pris en vue par les Latins.

Albert Baca, qui ètait déjả l'auteur d'un recueil de Selected Letters of Aeneas Silvits Piccolomini (Londres, 1969) a muni le texte d'annotations qui indiquent notamment les sources bibliques. La traduction est soigneuse, bien qu'il ne soit pas toujours aisé de rendre la concision du latin humaniste ( we agree in using the same words, but we are in conflict as to their meaning " pour "pax erit in nominibus, bellum in rebus»).

Pierre-François Moreau.

Jacques Gres-Gayer, Paris-Cantorbéry: le dossier d'un premier acuménisme (I7I7-1720). Préf. Robert Runcie. Panis, Beauchesne, 1989. 13,5 × 21,5, $576 \mathrm{p}$. ( (Théologie historique. Textes, dossiers, documents ", 13).

La crise précipitée par la bulle Unigenitus au sein de l'église gallicane ouvrit, de part et d'autre de la Manche, des espoirs dont cet ouvrage propose le récit documentaire. Pendant trois ans, de 1717 à 1720, William Wake, archevèque de Cantorbéry, échange une correspondance autour de l'idée d'un rapprochement des deux églises avec Louis Ellies Du Pin. Wake est entré en relations avec Du Pin par l'entremise de William Beauvoir, chapelain de l'ambassadeur d'Angleterre à Paris, qui le tient informé de l'évolution des affaires religieuses en France ${ }^{33}$. L'ensemble de la correspondance est reproduit, avec quelques documents de première importance, par J. Gres-Gayer, qui fournit en outre d'abondantes et érudites notes

33. Cet épisode connu des relations entre les anglicans et les jansénistes français a fait l'objet de deux études déjà anciennes: J. H. Lupton, Archbishop Wake and the Project of Union ( $1717-1720)$ between the Gallican and Anglican Churches, Londres, 1896, et Edmond Preclin, L'Union des églises gallicane et anglicane, Paris, 1928. 
explicatives. La présentation claire des documents, systématiquement résumés et situés, et qui figurent ici dans leur langue originale (principalement anglais, français et latin), permet de se déplacer sans jamais se perdre dans cette précieuse correspondance. Il faut, d'ailleurs, remercier l'auteur de la mettre ainsi à la disposition des chercheurs.

Pourtant cette présentation même, en dépit de son évidente commodité, appelle quelques réserves, ainsi que la lecture proposée dans la brève introduction qui précède les documents. J. Gres-Gayer a cru bon de diviser l'ensemble en cinq groupes majeurs, censés reproduire la progression des événements, des « préparations" et des "espérances" à l" "échec " et à la "dispersion ", en passant par une phase où l'on croit aller « vers le schisme». En créant ainsi l'impression trompeuse d'une succession d'étapes homogènes, c'est-à-dire en fait en persistant à reconstituer, contre l'arbitraire d'une sélection, la logique d'un récit, il sembie que J. Gres-Gayer cède à une illusion rétrospective, entretenue par son désir d'intégrer l'épisode décrit dans le cadre général de la problématique de l'œcuménisme. Or, si le projet a jamais eu une réalité à l'époque, ce fut avant tout dans l'esprit de Beauvoir, qui semble tout au long de ses lettres aveuglé par l'enthousiasme pour une idée en laquelle peu des intéressés, à commencer par Wake luimème, semblent croire. Il exista aussi, sans doute, dans l'esprit des adversaires catholiques du jansénisme, ou des adversaires anglicans du \& papisme ". Le même sentiment anticatholique qui rendait les jansénistes sympathiques aux yeux de certains, fit craindre à d'autres le retour du « complot papiste ", dont ce projet fantôme avait l'air d'être la forme la plus sournoise, partant la plus à redouter ${ }^{34}$.

Replacée dans la perspective longue de la vision anglaise du catholicisme, la correspondance présentée ici reprend des proportions plus sobres. L'unification de l'Église d'Angleterre et de l'Église de France fut certes un des serpents de mer de toute la période qui suivit la naissance du mouvement janséniste en France, puisque dès 1650 une tentative de rapprochement fut amorcée du côté anglais par Richard Stewart et sir George Ratcliffe, sans rencontrer aucun succes. En 1694, sir William Trumbull est averti par un correspondant qu'une réforme sur le modèle anglais se prépare en France. Entre les deux dates, la littérature janséniste est largement traduite en Angleterre, dans une série compacte de publications inaugurée par la traduction des Provinciales en 1657. L'épisode étudié par J. Gres-Gayer s'inscrit donc dans la continuité d'un intérêt prudent manifesté par les Anglais à l'égard de l'histoire de l'Église de France, en particulier dans ses rapports avec Rome $^{35}$. Quand Beauvoir cherche à convaincre Wake de la possibilité d'une ouverture, ce n'est donc pas la première fois qu'un rapprochement a été esquissé sans succès. La méfiance à l'égard du catholicisme est alors trop fermement

34. Les ennemis d'un rapprochement exagérèrent la portèe de la correspondance aussitôt qu'ils connurent son existence. On alla jusqu'à parler du « plus abominable complot qu'un Docteur Catholique ait pu tramer en matière de religion w (cite par Ruth Ci.AkK, Sirrangers and Sojourners al Port Royal, Cambridge, 1932, p. 258).

35. En 1680, Robert Boyle envisagea de faire traduire la préface du Nouveau Testament de Port Royal pour l'édition irlandaise du Nouveau Testament dont il organisait la publication. Il dut finalement accepter un compromis, de nombreuses autorités ecclésiastiques supportant mal qu'on ègratignả les figures les plus illustres de la Réforme anglicane. 
ancrée, en Angleterre, pour que de véritables espoirs soient permis. Si la critique de la puissance du pape et le refus de son infaillibilité peuvent sembler séduisants à des mentalités anglicanes, en revanche la distance théologique demeure irréductible sur bien des questions. L'attitude gallicane, même en ses aspects les plus extrêmes, est de toutes façons timorée aux yeux de qui garde en mémoire l'audace politique du schisme henricien. Enfin, l'organisation interne de l'église anglicane, préoccupée depuis le xvif siècle par la question de la comprehensiveness, ne permet pas d'envisager le rapprochement avec une église catholique sans risques pour l'équilibre déjà fortement menacé des différentes composantes de l'Église d'Angleterre. L'opposition entre low church et high church, le problème du sort des dissidents, la récente crise arienne empêchent d'envisager le rapprochement avec réalisme. Wake, au demeurant, ne semble jamais très convaincu du sérieux des possibilités décrites par certains de ses correspondants, qui montrent parfois trop d'empressement à vouloir faire revenir les anglicans dans le giron de l'église catholique. Il y a, dès le début, un malentendu qui ne se dissipe jamais vraiment et qui donne à cette correspondance l'apparence intermittente d'un dialogue de sourds. Relevons à ce propos l'étrange affirmation de l'auteur, pour qui Du Pin "ne se propose pas de réécrire» certains des Trente-neuf Articles de l'èglise anglicane, mais suggère " une rédaction plus conforme » (p. 29) ou veut " proposer des modifications textuelles $»$ (p. 33).

Enfin, à un niveau plus théorique, on pourrait reprocher à J. Gres-Gayer de séparer le théologique đu politique de façon excessive, alors que seule la prise en considération de leur solidarité parfois étroite permet de rendre compte des phénomènes religieux de la période, en particulier du côté anglais, où la critique des "superstitions papistes " n'est jamais séparable d'une critique du pouvoir temporel du pape, et où les débats sur la question trinitaire prennent immanquablement l'aspect d'une réflexion générale sur la notion d'autorité. J. Gres-Gayer a trop tendance à traiter le politique comme "élément " externe et souvent nuisible des relations entre les différentes organisations ecclésiastiques, ce qui l'amène à lui donner une place périphérique ${ }^{36}$. Or, un véritable renouvellement de l'étude des mouvements religieux n'est possible qu'à condition d'abandonner l'idée que le religieux disposerait, en théorie comme dans les faits, d'une autonomie constamment menacée par les empiétements du politique.

Jean-François Banlon.

36. Ajoutons en passant que certains jugements portés par l'auteur semblent parfois rapides: tandis que Du Pin a selon lui a un goût trop affirmé pour une érudition sans compromis" (p. 15), Law se trouve crédité d'un « merveilleux système monétaire » (p. 24). 
Pierre de Saint-Amand, Les Lois de l'hostilité. La politique à l'âge des Lumières. Av.-pr. de Michel Serres. Paris, Seuil, 1992. $14 \times 20,5,204$ p., index ( La couleur des idées »).

Cinq essais sur Montesquieu, Voltaire, Diderot, Rousseau, Sade constituent la trame de cet ouvrage. Comme point de convergence, une commune volonté d'oublier " les lois de l'hostilité ", c'est-à-dire d'exorciser la guerre, d'ignorer la violence. Dûment refoulée, cette "part maudite " du patrimoine des Lumières ressurgirait chez Rousseau et chez Sade. Pierre de Saint-Amand utilise à la fois, et au mème titre, des ceuvres de fiction, Lettres persanes, ou Contes de Voltaire, ou des traités philosophiques. Ainsi c'est Rica, le mondain des Lettres persanes qui est présenté comme porte-parole de l'auteur de l'Esprit des lois... Un projet d'anthropologie politique des Lumières sous-tend l'ensemble de ce discours. Le schéma est intéressant mais la démonstration n'emporte pas la conviction, du moins celle de l'historien. Comment la vision de la guerre serait-elle identique pour un contemporain du traité d'Utrecht et de l'abbé de Saint-Pierre et pour un écrivain qui traverse les guerres révolutionnaires?

Monique Cotret.

Louis AmIABle, Une Loge maçonnique d'avant 1789. La loge des Neuf Sceurs.

Réimpr. augm. d'un commentaire et de notes critiques de Charles PORSET. Paris, EDIMAF, 1989. $15 \times 22,5,399-239$ p.

Le livre de L. Amiable est un texte injustement méconnu - intéressant à la fois les spécialistes des XVII et $x^{e} x^{\mathrm{e}}$ siècles - que $\mathrm{C}$. Porset réédite et présente au lecteur contemporain. L'histoire de la Loge des Neuf Sneurs est l'ceuvre d'un important dignitaire de la franc-maçonnerie sous le Second Empire et la Troisième République, L. Amiable, qui fut avocat, magistrat et maire du Ve Arrondissement de Paris. Son livre, publié au lendemain de sa mort survenue en 1897 , constitue une monographie qui se voulait « une contribution a l'histoire intellectuelle de la société française d'Ancien Régime " (C. Porset) et une étude sur les causes de la Révolution. Pendant les dernières années du $x x^{2}$ siècle, en dépit des nombreuses manifestations républicaines, l'historiographie contre-révolutionnaire, conforté par la publication des Origines de la France contemporaine de Taine, conservait une certaine influence. La franc-maçonnerie était alors très souvent dénoncée par la droite, et son rôle mythifié - d'où une volonté des historiens républicains d'opérer une contre-attaque en recourant de manière plus rigoureuse aux sources originales. Gráce à un fonds d'archives aujourd"hui disparu, le livre de L. Amiable satisfait a cette exigence, tout en demeurant une " auvre de combat et de propagande ". Il montre que les maçons des Neuf Sceurs - loge créée en 1776, " fille posthume d'Helvétius s oú Voltaire se fit initier l'année même de sa mort et à laquelle sont associés les noms de l'astronome Lalande, de l'erudit Court de 
Gébelin, de Benjamin Franklin, de Dupaty et de Pastoret qui travaillèrent à la réforme de la législation criminelle - sans être véritablement des révolutionnaires, s'inscrivent dans le courant favorable à la monarchie constitutionnelle avant que la Loge ne connaisse une longue éclipse (et plusieurs deuils sous la Terreur) entre 1792 et 1806 et ne disparaisse définitivement, an lendemain de la révolution de 1848. La réimpression anastatique de l'édition de 1897 est enrichie par une introduction de C. Porset qui situe le travail de $\mathrm{L}$. Amiable dans une perspective historiographique très éclairante ainsi que par un ample commentaire critique ; celui-ci est nourri par des notices historiographiques minutieuses et par des mises au point bibliographiques d'une grande utilité, qui suivent pas à pas le texte de L. Amiable. L'appareil critique est complété par un index et par une table analytique des matières qui en facilitent la consultation. Dans l'abondante littérature consacrée à la franc-maçonnerie et au bicentenaire de la Révolution, on distinguera cet ouvrage qui ne propose pas seulement un bilan des études accomplies mais ouvre des perspectives de recherches nouvelles et stimulantes.

Chantal GreLl.

Paul Kléber Monon, Jacobitism and the English People, 1688-1788. Cambridge, Cambridge University Press, 1989. $16 \times 23,5,408$ p., bibliogr., index.

Le Xvir ${ }^{e}$ siècle, « période de confusion intellectuelle » $(p, 42)$ : voilà un démenti salutaire à la transparence et à la clarté dont on crédite trop aisément la centaine d'années qui s'étend de la Glorieuse Révolution d'Angleterre à la Révolution française. Encore que l'auteur demeure implicite sur ce plan, la périodisation adoptée est bien celle des Lumières triomphantes. De 1688 à 1788, P. Monod, jeune historien canadien, nous livre l'envers de l'Enlightenment et sa cause perdue : l'épopée des Stuarts qui, de Jacques II à Jacques III ou à Charles-Edouard, ne parviennent pas à reconquérir leurs royaumes.

Lorsqu'il s'enfuit de Londres en décembre 1688 pour laisser la place à Guillaume d'Orange, Jacques II ouvre une nouvelle page de l'histoire britannique, longtemps occultée ou confinée dans les marges celtiques d'Irlande et d'Écosse. Sans céder à ce qu'il appelle joliment l' " opium de la nostalgie réactionnaire », $P$. Monod relève dans sa thèse magistrale un double défi : il montre savamment que le jacobitisme joue un rôle au moins aussi important en Angleterre qu'en Écosse ou en Irlande; il souligne la convergence entre le loyalisme Stuart et la contestation sociale de l'ordre établi. Du jacobitisme aristocratique au jacobitisme populaire, quelles médiations?

Le jacobitisme, apparemment, est soluble dans l'alcool. Boire à la santé du Prétendant est l'un des rites bachiques en usage qui témoigne, par sa rémanence, d'une sentimentalité souvent bridée par ailleurs. Plus encore qu'un mouvement ou qu'une idéologie, le jacobitisme est une "culture $\%$, un "système de signes" (p. 7) auquel P. Monod tente d'appliquer la grammatologie de M. Derrida pour conclure benoitement que cela ne le mène á rien. Par contre, P. Monod se révèle 
un lecteur enthousiaste de Maurice Agulhon : les études de sociabilité sont parmi les plus novatrices pour saisir, par-delà les clivages trop marqués, l'ambiguîté $d u$ jacobitisme. « La culture politique jacobite nourrit une sociabilité qui n'est ni totalement conservatrice, ni totalement démocratique » (p. 292).

Fondamentalement, le jacobitisme constitue une réponse infructueuse à la " perte d'unité " qui marque le xviıl siècle anglais. Le loyalisme Stuart repose sur une foi quasi religieuse en un roi arbitre qui libère ses peuples des partis et factions qu'engendre la corruption parlementaire. Cette crainte d'une " désintégration morale " explique la résurgence massive du phénomène en temps de crise politique - en particulier lorsque l' « usurpateur" Georges de Hanovre devient roj d'Angleterre. Cette vision alternative du monde se taille également un franc succès dans les Belles Lettres comme dans la littérature périodique, dans la chanson comme dans l'iconographie. À bien des égards, le jacobitisme puise dans le vieux fonds de la culture populaire pour la remodeler à son tour. On lira de fort belles pages sur le mois de mai et les réjouissances de printemps qui encourent dès la première révolution anglaise la vindicte puritaine.

P. Monod ne croit apparemment pas à l'autonomie de la culture populaire. N'ignorant rien des travaux français (R. Mandrou) ou italiens (C. Ginzburg), P. Monod est attentif à l'existence de visions du monde, revivifiées par la culture jacobite, hantée par le souvenir de la révolution puritaine des années 1642-1660. Le jacobitisme engage une stratégie de la mémoire, ou plus précisément de la remémoration sélective: de Cromwell à Guillaume d'Orange, de Guillaume d'Orange à Georges $\mathrm{I}^{\mathrm{er}}$, de Georges $\mathrm{I}^{\text {er }}$ à Georges II, une rare continuité fantasmatique permet d'associer les figures diaboliques d'usurpateurs. $\AA$ l'inverse, le portrait du Prétendant emprunte au gente immémorial de la pastorale, on à la théologie des deux corps du roi. (L'importance de Kantorowicz est clairement soulignée.)

L'arrivée au pouvoir de Georges III en 1760 semble cependant mettre partiellement fin à cette mystique jacobite, ou du moins elle se marginalise. Il n'en est pas moins vrai que "bien qu'enivrant, le culte de la monarchie qui se développe sous Georges III est vide sur le plan théorique. Jusqu'à ce jour, la monarchie britannique n'a pas d'autre titre à faire valoir que la tradition » (p. 44).

Faut-il tenter un rapide bilan de ce travail en tous points remarquables? Il s'inscrit de toute évidence dans le renouvellement des études sur le jacobitisme auquel s'associent les noms d'Eveline Cruickshanks, de Jonathan Clark ou de Guy Chaussinand-Nogaret. L'exposition et le colloque qui se sont tenus en 1992 à Saint-Germain-en-Laye ont montré l'intérêt du public français pour cette page d'histoire européenne. La Revue de la Bibliothèque nationale a du reste publié $\left(n^{\circ} 46\right.$, hiver 1992), sous le titre « Les Jacobites ", les actes de cette manifestation à laquelle tant d'historiens se sont associes.

P. Monod parvient à écrire une histoire politique largement ouverte sur les questions culturelles, les phènomènes de sociabilité, l'anthropologie religieuse, voire la littérature. Cette histoire totale, en un mot, n'ignore aucun secteur de la vie des hommes du xvıı 'siècle anglais; elle témoigne également - ce qui est à l'honneur de l'école française - de remarquables convergences avec les recherches menées chez nous. L'histoire des mentalités et des comportements permet de lever l'hypothèque d'une approche trop strictement idéologique de la 
vic politique - sans sombrer dans le réductionnisme pour autant. On ne sera pas surpris de constater finalement la dette que P. Monod a contractée envers Marc Bloch : le toucher des écrouelles est abandonné par Guillaume d'Orange comme par les Hanovre - respectivement après 1688 et après 1714. Seuls les Stuarts (dont la reine Anne, qui règne de 1702 à 1714) ont encore recours à ce ressort thaumaturgique. Mais, nous dit malicieusement P. Monod, cette disparition n'est pas liée à la «diffusion du rationalisme mais à la nécessité politique » (p. 131). On ne peut que le féliciter pour sa brillante synthèse.

Bernard CotrRet.

Les Aventures du citoyen Jean Conan. Avanturio ar citoien Jean Conan a Voengamb. Texte breton original, trad. franç. inédite, présent. littéraire, linguistique et historique par Y.-B. Piriou, F. Favereau et J. Cornetre. Morlaix, Skol Vreizh, 1990. 21,5 × 30,5, 319 p., index, photogr.

Quel bel ouvrage riche de 7050 vers bretons traduits en français pour le plus grand plaisir des lecteurs qui ne connaitront point la langue de Jean Conan. Mais la présence de la langue maternelle rassure : le verbe de Jean Conan se nourrit d'elle pour faire de sa propre vie une épopée jusqu'à la fin de ses campagnes. Ensuite, jusqu'à la mort, il lui reste l'écriture ou plutôt la passion - née à l'abbaye de Beauport - de la rime, une façon de tout dire ou de tout transformer en poésie. Voilà le héros devenu héraut ou inversement... Remercions l'équipe de professeurs de breton, d'historiens et de photographes qui ont conjugue leurs talents, leurs recherches et leurs paysages pour ne rien laisser dans l'ombre du pays, de l'époque et du personnage de Jean Conan, tout en laissant quartier libre à son étonnante poésic qui est vie et paroles quotidiennes. Jean Conan a vingtcinq ans quand commence la Révolution. Depuis trois ans déjà, par chagrin d'amour, il s'est embarqué pour Terre-Neuve où il a fait naufrage en juin 1787. De retour au pays, il se marie avec Jacquette Menguy dont il a un fils, Jean-Marie, qui meurt en 1792. Cette année-là, enrôlé sous le matricule 2158, il participe, le 10 aoùt, à la prise des Tuileries. L'année suivante est remplie par la guerre et 1794 commence de même : le 26 juin, il est à Fleurus. Mais sa carrière militaire s'interrompt quelques jours plus tard : l'écroulement d'un mur lui meurtrit le dos et fait de lui un soldat bon pour la réforme. Désormais, il ne quitte plus la Bretagne. Il s'y remarie en 1797 à Marie-Jeanne Le Thomet après la naissance de leur premier enfant et son récent veuvage. Il y guerroye contre les Chouans dans les dernières années du xvir siècle et s'installe comme tisserand à Guingamp. Le jour, il travaille pour nourrir les siens; la nuit, il se donne tout entier à son ceuvre et aux dessins qui l'illustrent, Sur les sept manuscrits qu'il a légués, seuls quatre ont été retrouvés. Parmi eux, des vies de saints bretons et les a aventures du citoyen Jean Conan de Guingamp \%. Elles suivent la trame de sa vie de matelot-soldat et la restituent, vers par vers, dans ses moindres détails tant la mémoire de Jean Conan épouse le rythme des rimes bretonnes. C'est ce chant intérieur qui fait de ce récit 
l'épopée d'un homme du peuple. Jean Conan en attendait beaucoup et il n'avait pas tort :

" Mais le fait d'être un pauvre homme ne me tracasse pas;

Après moi, mon nom me survivra sur le papier.

Quelqu'un, peut-être un jour, après avoir lu ce cahier

Dira : «Que Dieu pardonne à celui qui l'a écrit!»

Anne-Marie CoculA.

Rémy CazAls, Autour de la Montagne Noire au temps de la Révolution: 17741799. Carcassonne, Clef 89 Aude, 1989. $14 \times 22,198$ p., ill., bibliogr., index.

Appuyé sur une solide connaissance des archives locales, ce petit livre compose au fil des années et des lieux le kaléidoscope de la vie économique, sociale et culturelle d'une terre languedocienne dans le dernier quart du xvir" siècle, articulant toujours l'événement local aux enjeux généraux de la période. La Montagne noire: des hautes terres d'accès difficile, sensibles aux crises de subsistances, bien éloignées de l'autorité royale personnifiée par l'intendant de Montpellier. Mazamet et Carcassonne : deux centres lainiers confrontés ả la crise de la draperie languedocienne, et pour lesquels la route et l'accès au Canal royal sont des enjeux vitaux. Terre protestante, la règion est aussi le thêatre de l'affaire Sirven (condamné comme Calas), et un foyer des Lumières, avec l'École de Sorèze. L'auteur a ainsi composé, d'une plume éndite, une manière de guide pour le voyage audois.

Philippe Minard.

Roberta Curiel, Bernard Dov Cooperman, Le Ghetto de Venise. Trad. de l'anglais Jean-Pierre BArDos, photogr. de Graziano ARIC. Paris, Herscher, 1990. $24 \times$ 28,176 p., ill. en noir et en coul.

L'abondante bibliographie consacrée à l'histoire de la communauté juive vếnitienne, à celle de son quartier, le ghetto, crée en 1516, s'errichit done d'un ouvrage supplémentaire. Il ne s'agit pourtant pas simplement d'un ouvrage de plus. La conception, l'écriture, la richesse iconographique de ce livre en définissent clairement les destinataires : un public cultivé davantage que le cercle des « spécialistes ». Amateurs de Venise, curieux de l'histoire de ce qui fut le premier ghetto pourront donc partager ensemble cette promenade.

Le premier texte (la création du ghetto de Venise) met en place la chronologie et les faits. Il retrace l'histoire spatiale et ethnique du quartier, des premières 
décennies du $x^{\mathrm{e}}$ siècle à 1797 . En 1516 , le Sénat cantonne les juifs dans l'îlot de la nouvelle fonderie (ghetto nuovo). En juillet 1797, un décret de Bonaparte émancipe les juifs. Les portes du ghetto sont abattues, leurs clefs sont exhibees, un arbre de la Liberté est planté. Avec le traité đe Campo Formio, la Vénétie passe sous domination autrichienne. La discrimination spatiale n'est cependant pas restaurée. Entre ces deux repères, l'étude de la communauté juive, ou plutôt des communautés, et des diffèrents flux d'immigration des «Levantins » et des «Ponantais" qui viennent grossir les premiers groupes des Italiani et des Tedes$c h i$, nourrit l'examen social et morphologique du ghetto.

Dès lors, le livre est bien conçu comme une promenade. Découverte du ghetto nuovo d'abord, puisque ce périmètre de $7000 \mathrm{~m}^{2}$ fut le premier assigné à la résidence obligatoire de la population juive. L'analyse monumentale des trois synagogues du campo du ghetto nuovo (scuola grande tedesca, scuola canton, scuola italiana), jusqu'à leurs dernières et récentes restaurations, suit. Un bref intermède, consacré à la vie sur cette petite place, rompt toutefois la succession de ces trois monographies. L'analyse du ghetto vecchio, réservé en 1541 aux juifs levantins quand la surpopulation du ghetto nuovo fut devenue extrême, obéit à la même logique descriptive. Après quelques remarques toponymiques et architecturales sur la zone du ghetto vecchio, deux chapitres sont rèservés à la scuola grande spagnola et à la scuola levantina. Le parcours se clôt avec le ghetto nuovissimo, créé en mars 1633, à la demande des Levantins et des Ponantais pour y loger les plus riches des derniers immigrés séfarades.

La structure narrative va donc, à l'égal d'une visite, des espaces du dehors à ceux du dedans, des places et des ruelles aux synagogues. Et le visiteur, au terme du livre, abandonne le ghetto et les extrémités du quartier de Cannaregio pour s'embarquer pour le cordon littoral de San Nicolo du lido, où depuis 1386, après accord de la seigneurie de Venise, les juifs disposent d'un cimetière. Bousculant quelque peu la logique géographique, le trajet comporte un dernier retour au ghetto pour une présentation du musée d'art juif et du catalogue des pièces exposées. Une bonne bibliographie, un glossaire et un index complètent la succession de ces diverses études.

Le livre décrit et montre. Le texte, du début à la fin, s'appuie sur une illustration splendide qui sait délaisser les images habituelles. C'est donc bien, on le répétera, un parcours qui est proposé, dans l'espace, dans le temps, même si la chronologie, souvent, paraît quelque peu aplatie. On pourra regretter que les anecdotes ou les petits faits soient trop privilégiés aux dépens d'une véritable analyse sociale, économique et culturelle de la communauté juive dans les trois derniers siècles de la République. On relèvera que le premier chapitre, à vocation davantage historique, est trop laconique, en dépit d'excellentes études récentes, sur la situation des juifs dans la ville et dans l'Empire des Vénitiens avant que le cantonnement spatial ne soit instauré. L'exposé aurait de même gagné à mieux préciser comment évoluent, dans l'Italie des dernières décennies du $\mathrm{xv}^{\mathrm{t}}$ siècle et des premières années du $x^{e}{ }^{e}$ siècle, les représentations à l'égard des juifs. Sans doute aussi l'analyse des transformations sociales et spatiales qui modifient Venise durant cette même période aurait-elle contribué à replacer la creation du ghetto dans un mouvement général de recomposition des équilibres urbains. Non pas que ce chapitre ne fournisse pas quelques éléments d'explication ou de comparai- 
son. Mais la référence plus systématique à des études, citées par ailleurs dans la bibliographie, aurait enrichi un exposé, parfois rapide et linéaire. Lorsque, dans le reste de l'ouvrage, le parti pris de la visite s'affirme avec plus de vigueur, le lecteur cède avec moins de réticences au plaisir de la promenade et des images.

Élisabeth Crouzet-Pavan.

Kurze Geschichte Siebenbürgens. Hrsg. von Béla KÖPECzI, unter Mitarbeit von Gábor Barta, István BónA, László MaKkai, Zoltán Szász, Redaktion der deutschen Ausgabe Zoltán Szász. Budapest, Akadémiai Kiadó, 1990. $17 \times$ 24,4, 781 p., bibliogr., chronol., index.

Voici non pas une brève histoire de la Transylvanie, mais la traduction allemande de l'abrégé de l'Histoire de Transylvanie publiée en 1986 par l'Académie des sciences hongroise ${ }^{37}$. La traduction française de ce même abrégé est désormais parue (Budapest, Akadémiai Kiadó, 1992). Ces publications en différentes langues reprennent une tradition bien établie : l'histoire de la Transylvanie s'est toujours écrite en hongrois, en allemand, en roumain et ses historiens n'ont eu de cesse de s'adresser à la communauté internationale par l'intermédiaire du latin et de l'allemand, puis du français et de l'anglais.

Pour une Histoire abregée, les appareils critique et cartographique restent considérables. L'ouvrage se clôt sur un excellent index des noms de lieux (avec leurs équivalents dans les différentes langues), des noms de personnes et des principales notions. Une chronologie fournit au lecteur les repères nécessaires. Vingtsix cartes permettent non seulement de visualiser, dans l'espace et dans le temps, l'installation, puis la répartition géographique des diffèrents peuples de la Transylvanie, mais aussi la diffusion de leurs cultures et de leurs religions et, enfin, par la présentation de l'économie, des divisions administratives et des projets de rectification de frontières, les liens de la Transylvanie avec les États et provinces voisins. La bibliographie a été actualisée d'une édition à l'autre : somme sans exclusive des travaux consacrés à la Transylvanie, elle est avant tout une bibliographie raisonnée qui fait systématiquement le point sur les diffèrentes thèses en présence, de ce fait utile au spécialiste comme au lecteur curieux.

Les auteurs de l'Histoire en trois volumes ont eux-mèmes assuré le travail de synthèse. Aussi l'économie interne de l'ouvrage n'a-t-elle tien perdu de sa cohérence $^{38}$. Béla Köpeczi a rédigé l'introduction et le bref aperçu qui va du traité de Trianon (1919) à la fin de la Deuxième Guerre mondiale, terme de cet ouvrage.

37. Pour l'abrégé : Erdély rövid története, Budapest, Akadejniał Kiadó, 1989 et pour l'édition en trois volumes : Erdély története, Béla Kópeczi, Andras Mocsy, Lászlo MAKkat, Zoltán SzÁsz, èd., 3 vol., Budapest, Akadémiai Kiadó, 1986.

38. L'Histoire en trois volumes et l'Abrégé constituent cependant deux ouvrages distincts. Les auteurs ont en effet tenu compte des critiques et suggestions adressées an premier ouvrage. Cf. Tanulmanyok Erdély történetéröl. Szakmai konferencia Debrecenben, 9-10 octobre 1987 (Entretiens et conférences sur l'Histoire de Transylyanie), István RAcZ, éd., Debrecen, Csokonai Kiadó, 1988, avec un résumé en anglais. 
István Bóna a assuré la rédaction de la première partie «de l'Antiquité à la conquête hongroise " (895); le même a encore étudié, en seconde partie, la "vie commune hungaro-slave (895-1172) ». Toujours pour la seconde partie, consacrée à la Transylvanie dans la Hongrie médiévale, László Makkai a traité la formation de la société d'ordres (1172-1526). Gábor Barta, Katalin Péter et Agnes R. Várkonyi se sont partagé la troisième partie ou l'étude de la Principauté de Transylvanie (1526-1711). Enfin, Zsolt Trócsányi, Ambrus Miskolczy et Zoltán Szász ont étudié dans la quatrième et la cinquième partie l'histoire de la Transylvanie dans l'Empire des Habsbourg (1711-1918). Depuis l'Histoire de Transylvanie de László Makkai, parue successivement en hongrois et en français ${ }^{39}$, le sujet était resté l'apanage des historiens roumains ${ }^{40}$. Cette fois, l'équipe est exclusivement composée de chercheurs hongrois, tous spécialistes du domaine traité, mais l'ambition d'ouvrir la voie à une collaboration renouvelée entre historiens hongrois et roumains n'en est pas moins rappelée.

Ce remarquable instrument de travail n'est-il cependant qu'une histoire hongroise de la Transylvanie, voire une " falsification délibérée », comme l'ont affirmé les historiens roumains - ou comme ils ont été contraints de l'affirmer, notamment en 1987, quand la délégation roumaine n'a pas obtenu l'autorisation de se rendre au Colloque international des Lumières qui, cette année-là, se tenait précisément à Budapest ${ }^{41}$ ? Il est vrai que la situation géographique de la Transylvanie historique $^{42}$ et la chronologie politique, qui sert d'armature à cette Histoire, compliquent infiniment la tâche des historiens. Les principes et la méthode qui caractérisaient l'Histoire en trois volumes ont été cependant réaffirmés avec force, ils doivent également constituer la pierre angulaire de ce renouveau tant attendu de l'histoire de Transylvanie.

Le texte s'appuie donc directement sur les documents, archives inédites ou collections de sources, résultats des recherches archéologiques les plus récentes. D'où la brièveté de l'aperçu 1919-1947 que Béla Köpeczi justifie par l'impossibilité d'accéder aux archives. Les auteurs souhaitaient aborder le plus librement possible le passé de cette région âprement disputée. Ils réexaminent donc systématiquement les principaux points de désaccord entre les historiographies hongroises et roumaines ${ }^{43}$. L'hypothèse de la continuité daco-roumaine ou de la fusion des cultures dace et romaine, dans laquelle les historiens roumains voient la naissance de la nation roumaine, est écartée par une démonstration qui, à partir

39. L. MaKkal, Erdély története, Budapest, 1944 et Histoire de Transylvanie, Paris, 1946.

40. $\AA$ titre de comparaison, en français : Brève histoire de la Transylvanie, Constantin Daiconiciu, Miron Constantinescu, éd., Bucarest, Ed. de l'Académie de la République socialiste de Roumanie, 1965.

41. Synthèse des critiques roumaines : Stephan PAscu, Mircea Musat, Florin Constant1NIU, “ Bewusste Fälschung der Geschichte unter der Ägide der Ungarischen Akademie der Wissenschaften ", Forschungen zur Volks- und Landeskunde, t. 30, 1987, p. 11-36.

42. La Transylvanie historique est plus petite que la province roumaine qui porte aujourd'hui le nom de Transylvanie. Elle est limitée au nord et à l'est par la chaine des Carpates, au sud par les Alpes de Transylvanie et domine à l'ouest la grande plaine hongroise.

43. Bonne synthèse des thèses roumaines, of. Cornelia Bodea, Virgil CandeA, Transylvania in the History of the Romanians, Boulder/New York, East European monographs/ Columbia University Press, 1982. 
de fouilles récentes et d'une étude onomastique, essaie de prouver la disparition du peuple dace à l'époque des grandes invasions. Un autre temps fort de l'ouvrage est consacré à l'origine de la voĩvodie, division administrative calquée sur le comitat hongrois et non principauté aristocratique du type de la voïvodie slave. Sont encore discutés l'origine des Sicules, cette autre " nation " de Transylvanie, composée de guerriers nobles défendant la Frontière, le rôle, entre Habsbourgs et Ottomans, de la Principauté indépendante de Transylvanie, les routes économiques et donc les liens de dépendance plus ou moins étroits avec les États voisins, le poids démographique et économique, aux différentes époques, de chacune des " nations " transylvaines, les causes du rattachement en 1919 de la Transylvanie à la Roumanie. Aussi les éditeurs n'ont-ils en aucun cas cherché à effacer les contradictions qui séparent les différents collaborateurs de cet Abrégé. Ainsi István Bóna conclut-il à la pénétration progressive des Hongrois en Transylvanie à partir du $\mathrm{Ix}^{\mathrm{e}}$ siècle, alors que László Makkai maintient la thèse plus ancienne de l'invasion hongroise au début du XII siècle. Le lecteur, surtout non initié, se doit donc parfois de résister au doute méthodique qui ne peut manquer de le prendre à la lecture de cet ouvrage et au vertige des complexités entrevues.

Panorama complet de l'histoire de la Transylvanie des origines à nos jours, l'ouvrage n'en est pas moins sous-tendu par un fil directeur : " considérer également les caractéristiques des trois principaux peuples qui composent la Transylvanie d'un point de vue économique, social, politique ou culturel » et mesurer leur rôle respectif dans la construction d'une a entité " qui n'est ni vraiment un État, ni une nation, mais un tout, étroitement intégré dans un espace géographique et politique. En ce sens, cet Abrégé n'est pas écrit dans la perspective d'une seule nation, fût-elle dominée ou dominante, même si mille ans d'histoire commune (point de vue hongrois) sont censés faire pièce au droit du premier oocupant (point de vue roumain). En l'occurrence, il paraît vain de s'interroger sur les finalités de l'ouvrage ou de polémiquer pour savoir si tel ou tel aspect est fondé ou non. Dans ce modèle d'histoire à trois voix, tout se passe comme s'il fallait précisément se placer sous le signe des nationalités pour étudier la construction d'une entité plurinationale. L'ouvrage pose finalement une seule question : comment écrire l'histoire d'un creuset de peuples et de cultures déchirés entre plusieurs États-nations?

Christine Lebeau.

Correspondance entre Charles Andler et Lucien Herr, 1891-1926. Éd. établie, présentée et annotée par Antoinette BluM, préf. de Christophe Charle. Paris, Presses de l'École normale supérieure, 1992. 15,5 × 24, 298 p., bibliogr., index.

La publication de cette correspondance entre Andler (1866-1933) et Herr (1864-1926) comble une lacune de 1'histoire de la culture française. En effet, ces 
deux Alsaciens, partageant une commune formation de normalien (l'un est philosophe, l'autre germaniste) et des convictions socialistes ont profondément marqué leur époque. L'échange très spirituel et souvent amical regorge d'intelligence. Histoire des universités allemande et française, développement de la germanistique en France, réflexion sur la philosophie allemande, rien ne manque. C'est tout un pan de la culture savante de l'Europe qui se lit tout en complicité et générosité.

Le profond exégète de Nietzsche du Collège de France et le bibliothécaire et mentor de la rue d'Ulm sont ici en liberté : une fragrance de classe intellectuelle et un réel plaisir devant tant de savoir envahit le lecteur dès les premières lettres. D'autant plus que les notes précises et érudites ajoutent encore à une très riche moisson. Indiquons, en témoignage de gratitude pour cette lecture, une lettre de Lucien Herr à Carl Heinrich Becker du 19 juin 1925, en français et en allemand, dans les papiers de ce savant ministre de la République de Weimar (Nachlass C. H. Becker, SPKB, Berlin).

Dominique BoureL.

Jacques Girault, Benoit Frachon, communiste et syndicaliste. Paris, Presses de la Fondation nationale des sciences politiques, $1989.13,5 \times 22,364 \mathrm{p}$., index.

L'histoire du communisme ne profite pas encore de tous les bienfaits d'une glasnost qui semble bien tarder du côté des archives du Parti communiste français. Jacques Girault en aura visiblement souffert. Son ambition - écrire le plus rigonreusement possible une biographie politique d'une des plus hautes figures du communisme français - s'est heurtée bien souvent à l'absence de sources. Que de questions laissées sans réponses! La scrupuleuse honnêteté de l'auteur l'a conduit à parsemer son ouvrage de multiples points d'interrogation qu'il faudra bien un jour effacer. Le temps semble enfin venu d'écrire une histoire du mouvement ouvrier où les passions politiques le laissent à la rigueur de l'historien. Quoi qu'il en coûte encore à quelques-uns.

En dépit de ces obstacles, cette biographie retrace avec soin l'itinéraire politique et syndical de Benoît Frachon. Né en 1893 à Chambon-Feugerolles dans un milieu de mineurs, affecté spécial en 1914, ce metallo manifesta d'abord un tempérament libertaire, plus enclin à l'action syndicale qu'à l'engagement dans un parti politique. Séduit par la révolution bolchevique à l'esprit de laquelle il ne cessa d'être fidèle, il adhéra au Parti communiste tout en assumant des responsabilités syndicales régionales dans le cadre de la C.G.T.U. de la Loire. Depuis la scission syndicale de 1921, il se persuada très vite de la nécessité de l'unité syndicale à laquelle il resta attaché toute sa vie durant, malgré toutes les vicissitudes de l'histoire du syndicalisme français. Farouchement hostile au « téformisme ", il ne confondait pourtant nullement les ouvriers socialistes et les dirigeants de la S.F.I.O. - il sabla le champagne après avoir appris la mort de Blum - - de mème qu'il prétendit toujours privilégier une "union à la base " avant même toute unité syndicale organique. Toujours soucieux d'unité, il accepta de démis- 
sionner du Bureau politique et du Comité central du Parti communiste pour remplir ses fonctions de membre du Bureau confédéral de la C.G.T. réunifiée au Congrès de Toulouse en mars 1936 tout en conservant d'ailleurs, au sein de l'appareil communiste, d'importantes fonctions officieuses.

B. Frachon resta d'abord un militant communiste, conscient toutefois de l'importance d'une action syndicale autonome. L'articulation n'est pas aisée et les sources disponibles ne permettent pas toujours de comprendre comment celle-ci put se faire ni surtout comment il l'assuma. Au fil des années, B. Frachon apparait de plus en plus comme un homme de l'appareil communiste, rigide sans être sectaire nous assure J. Girault, qui n'accepte aucune critique sur le Parti ou l'Union soviétique émanant d'un non-communiste.

Une réserve, enfin, pourra être émise sur le mode d'approche délibérément retenu par J. Girault. Le livre est une biographie politique de Benoît Frachon. Les aspects personnels de sa vie ont été exclus. Selon l'auteur, il ne semble pas - au regard de l'histoire en tout cas - qu'un rapprochement vie privée/vie publique puisse être riche de sens. Ces deux facettes se trouvent-elles donc en si parfaite harmonie ? N'existait-il vraiment aucune discordance dont on aurait pu tirer quelque signification? Dès lors, si B. Frachon, syndicaliste et homme politique, ne se distingue nullement de B. Frachon inséré dans sa sphère sociale " privée ", écrire une biographie du secrétaire général de la C.G.T. ne se réduisait-il pas à porter un regard singulier sur une cinquantaine d'années d'histoire syndicale française? Et faut-il alors conclure que la proximité des dirigeants communistes et de leur appareil, voire leur identification à celui-ci sont telles qu'elles excluent toute approche biographique? On peut en douter à la lecture même des trop rares passages que J. Girault consacre à la personnalité de B. Frachon. Les pages où sont abordées ses méthodes de travail, sa vie clandestine ou sa culture sont riches d'enseignements.

Entre autres mérites, cet ouvrage soulève quelques bonnes questions de méthode. En France, l'histoire du mouvement ouvrier, dans le sillage de l'histoire sociale, a peu pratiqué le genre biographique. Jaurès, Vaillant, Delesalle, Thorez ont eu leur biographe. Beaucoup le cherchent encore. Et, en vérité, il n'est pas certain que ce mode d'approche soit le plus approprié à un effort de compréhension du rôle joué par un individu dans un milieu ou un réseau qui seuls le définissent socialement. Un militant n'est ni un écrivain ni un homme d'État. Sa façon de marquer l'histoire lui est bien propre. Son individualité ne prend une consistance historique qu'insérée dans un cadre social. C'est le jeu individu/milieu que l'historien doit saisir. C'est ce jeu-là qui fait l'histoire. La biographie reste-t-elle donc un genre encore possible? 